\title{
LARGE EDDY SIMULATION OF A TURBULENT SWIRLING COAXIAL JET
}

\author{
K.K.J.Ranga Dinesh ${ }^{1}$, A.M.Savill ${ }^{1}$, K.W.Jenkins ${ }^{1}$, M.P.Kirkpatrick ${ }^{2}$ \\ 1. School of Engineering, Cranfield University, Cranfield, Bedford, MK43 0AL, UK \\ 2. School of Aerospace, Mechanical and Mechatronic Engineering, The University of \\ Sydney, NSW 2006, Australia \\ Corresponding author: Ranga.Dinesh@Cranfield.ac.uk \\ Tel: +44 (0) 1234750111 ext 5350
}

Revised Manuscript Prepared for the Journal of Progress in Computational Fluid Dynamics

$07^{\text {th }}$ July 2009 


\title{
LARGE EDDY SIMULATION OF A TURBULENT SWIRLING COAXIAL JET
}

\begin{abstract}
Computational modelling of scalar mixing (both passive and active) is important in many ways as it can provide useful details for practical engineering problems which cannot be discovered easily from physical experiments. There is currently particular interest in jet mixing especially for environmental impact consideration. The present work uses the Large Eddy Simulation (LES) technique to study velocity and passive scalar mixing along with intermittency of a spatially evolving turbulent coaxial swirl jet. This approach computes the temporal development of large-scale flow structures by solving the transport equations for the spatially filtered mass, momentum and passive scalar. The finite volume technique applied on a Cartesian mesh is used for numerical discretisation. All the simulations have been carried out for sufficiently long time periods to ensure convergence of time averaged statistics calculated for both velocity and scalar fields. The simulations captured the potential core and also predicted high level turbulence intensities in the inner mixing regions. The probability density functions (PDF) and radial intermittency plots revealed an intermittent mixing behaviour especially in the outer region of the flow where the fluctuations of velocity rapidly change from rotational to irrotational and vice versa. The PDF and radial intermittency profiles exhibit Gaussian and non-Gaussian distributions close to the jet centreline and away from the centreline respectively.
\end{abstract}

Key Words: Coaxial jet, Swirl, Passive scalar mixing, intermittency, LES 


\section{INTRODUCTION}

Coaxial jets with swirl are commonly found in various engineering applications and remain one of the challenging problems in fluids mechanics and combustion applications. Swirl is generally used to stabilise the flow by enhancing mixing and hence helps to stabilise the flame in combustion systems. In combustion applications, swirl-induced recirculation can reduce the flame length and hence reduce the combustor length and cost. Depending on the strength of the swirl, recirculation zones and vortex breakdown (VB) can be formed in swirl stabilised flames. The formation of large scale coherent structure seen in swirl jets is responsible for instability in isothermal flows, and thermoacoustic instability in the combustion systems. There is thus a need for more research at both fundamental and design levels to improve the overall understanding of swirl based mechanisms, which can be effectively used to aid the design process in practical engineering applications.

During the past five decades, a number of theoretical and experimental studies have been carried out aiming to identify characteristics of swirling coaxial jets for various configurations including both unconfined and confined geometries. Ribeiro and Whitelow (1980) studied a coaxial jet with and without swirl while Roback and Johnson (1983) conducted experimental investigation for confined swirling coaxial jet and measured data for both the velocity and passive scalar fields. Gouldin et al. (1985) also investigated a coaxial swirl jet and discussed the velocity field characteristics, while Mattingly and Oates (1986) examined the mixing of coannular swirling flows. Billant et al. (1998) also studied the vortex breakdown and recirculation motion of swirling jets. Champagne and Kromat (2000) also studied the recirculation in swirling coaxial jets, while Wicker and Eaton (2001) conducted swirl 
effects on particles in coaxial swirl jets. Giannadakis et al. (2008) recently examined the influence of swirl on coaxial flow and discussed various results in addition to previous experimental findings.

Computational modelling of swirl induced coaxial jets has complemented the experimental work and now acts as a useful tool to resolve multiscale flow problems covering a wide range of length and time scales. Reynolds Averaged Navier-Stokes (RANS), Large Eddy Simulation (LES) and Direct Numerical Simulation (DNS) are three well known techniques used for such turbulent flow analysis. Out of the three techniques, LES is capable of capturing the unsteady three dimensional nature of turbulence with affordable computing power.

LES is a particularly useful technique to study high Reynolds number complex swirl induced flow configurations due to their inherent unsteadiness and three dimensional structures. Several groups have done state-of-art LES investigations for isothermal, combustion, sprays flows that involves swirl. For example, Akselvoll and Moin (1996) first computed mixing of turbulent confined coaxial jets using LES and discussed key important aspects such as shear layer distributions, pressure field and also compared results with the experimental data. Pierce and Moin (1998) subsequently carried out a LES calculation of a confined coaxial swirl jet with heat release and studied the combustion process. Grinstein et al. (2002) simulated unsteady non-reacting swirling flows in a fuel injector nozzle using the monotone integrated large eddy simulation (MILES) technique. Apte el al. (2003) also conducted LES calculations for a confined swirl flow configuration with particle simulations. Wang et al. (2004) performed LES of confined swirling flows and obtained encouraging 
predictions for $\mathrm{VB}$, recirculation zones, and coherent structures of single swirling jets. Lu et al. (2005) carried out a LES of turbulent swirling flows in a dump combustor and discussed the VB phenomena, shear layer instability, and vortico-acoustic interactions. Dianat, et al. (2006) also studied the scalar mixing of a confined coaxial jet using LES and discussed different numerical schemes for the discretisation of scalar transport equation. Garcia-Villalba et al. (2006) considered the large scale coherent structures in the near field of confined coannular swirl jet. Wegner et al. (2007) studied the flow and mixing fields in a generic combustor using LES and discussed the flow features occurs in more generic combustor, while Wang et al. (2007) performed a series of calculations for a gas turbine swirl injector using LES and discussed various flow aspects in great detail.

The intermittency behaviour of turbulent free shear flows also plays an important role since it influences many engineering applications including aeroacoustics, mixing, propulsion, and combustion. In general, flowfield intermittency makes a round jet exhibit different structure in both the transitional and turbulent regions. A few attempts have been made to study the intermittency of inhomogeneous free shear flows using classical RANS. For example, a $k-\varepsilon$ based intermittency model was developed by Byggstoyl and Kollomann (1981). Kollmann and Janicka (1982) studied the intermittency using a transport probability density function (PDF) approach. Cho and Chung (1992) also developed a more economical intermittency model by incorporating an intermittency transport equation into an already existing $k-\varepsilon$ turbulence model. 
The present work aims to study the flow field and mixing of an unconfined coaxial swirl jet using LES. The configuration considered here is a scaled version of a typical RB211 engine exhaust using the engine characteristics provided by Garner et al. (1997). The aim was to study the velocity field, passive scalar mixing and their intermittent behaviour for such a coaxial swirl jet. The outcome of this work will be eventually used to further analyse the hot gas jet for emission distributions. Here we discuss transient and time averaged quantities for both velocity and passive scalar fields together with the corresponding probability density functions and intermittency profiles. The remainder of the paper is organised as follows: in section II, we will discuss the governing equations and modelling. In section III, we will give a detailed description of the numerical setup for both simulations. Section IV will discuss the results from these simulations for both velocity and passive scalar fields. Finally, we end the paper with conclusions in section $\mathrm{V}$.

\section{GOVERNING EQUATIONS}

In LES the large-scale energy containing scales of motion are resolved numerically while the small, unresolved scales and their interactions with the large scales are modelled. The grid-filtering operator, known as a spatial filter, is applied to decompose the resolved and sub-grid scales in the computational domain. Applying the spatial box filter to incompressible governing equations, we obtain the filtered continuity, momentum and passive scalar equations for the large-scale motion as follows:

$$
\begin{aligned}
& \frac{\partial \bar{u}_{j}}{\partial x_{j}}=0, \\
& \frac{\partial \bar{u}_{i}}{\partial t}+\frac{\partial\left(\bar{u}_{i} \bar{u}_{j}\right)}{\partial x_{j}}=-\frac{1}{\rho} \frac{\partial \bar{P}}{\partial x_{i}}+\frac{\partial\left(2 v \bar{S}_{i j}\right)}{\partial x_{j}}+\frac{\partial\left(\tau_{i j}\right)}{\partial x_{j}},
\end{aligned}
$$


$\frac{\partial \bar{c}}{\partial t}+\frac{\partial\left(\bar{u}_{j} \bar{c}\right)}{\partial x_{j}}=\left(\frac{v}{\sigma}+\frac{v_{t}}{\sigma_{t}}\right) \frac{\partial^{2} \bar{c}}{\partial x_{j} \partial x_{j}}$

Where $u_{i}, \rho, p, v, c, \sigma$ and $\sigma_{t}$ denote the velocity, density, pressure, kinematic viscosity, passive scalar concentration, laminar and turbulent Schmidt numbers, and the strain rate tensor, $S_{i j}=\frac{1}{2}\left(\frac{\partial \bar{u}_{i}}{\partial x_{j}}+\frac{\partial \bar{u}_{j}}{\partial x_{i}}\right)$. The last term of equation (2) represents the sub-grid scale (SGS) contribution to the momentum and it is known as the SGS stress tensor. Hence subsequent modelling is required for $\tau_{i j}=\left(\bar{u}_{i} u_{j}-\bar{u}_{i} \bar{u}_{j}\right)$ to close the system of equations.

The Smagorinsky (1963) eddy viscosity model is used here to model the SGS stress tensor $\tau_{i j}=\left({\overline{u_{i}}}_{j}-\bar{u}_{i} \bar{u}_{j}\right)$ such that

$$
\tau_{i j}-\frac{1}{3} \delta_{i j} \tau_{k k}=-2 v_{s g s} \bar{S}_{i j}
$$

Here the eddy viscosity $v_{s g s}$ is a function of the filter size and strain rate

$$
v_{s g s}=C_{s} \bar{\Delta}^{2}|\bar{S}|
$$

Where $C_{s}$ is a Smagorinsky (1963) model parameter and $|\bar{S}|=\left(2 \bar{S}_{i j} \bar{S}_{i j}\right)^{\frac{1}{2}}$. The Smagorinsky model parameter $C_{s}$ appears in the model equation (5) is dynamically calculated using the localised dynamic procedure of Piomelli and Liu (1991).

\section{NUMERICAL TECHNIQUES}

A. Computational domain, flow conditions and grid resolution 
The coaxial swirl jet has a core jet with a diameter $D_{c}=0.878 m$ which is surrounded by an annular secondary jet with diameter $D_{a}=1.422 \mathrm{~m}$. The centre of the core jet is taken as the geometric centre line of the flow where $r=0$ and $x=0$. Two independent bulk axial velocities are used to describe the inlet velocities for the simulations, the bulk axial velocity of the core jet, $U_{c}=158 \mathrm{~m} / \mathrm{s}$, the bulk axial velocity of the secondary annular inlet, $U_{a}=104 \mathrm{~m} / \mathrm{s}$. The swirl velocity is introduced into the annular jet at exit with the swirl number defined as the ratio between the axial flux of the swirl momentum, $G_{\phi}\left(\mathrm{kgm}^{2} \mathrm{~s}^{-2}\right)$, to the axial flux of the axial momentum $G_{x}$ $\left(\mathrm{kgm}^{2} \mathrm{~s}^{-2}\right)$ multiplied by a characteristic length $R(\mathrm{~m})$. Here we take the radius of the swirl annulus as the characteristic radius. The swirl number is given by

$$
S=\frac{G_{\phi}}{R G_{x}}=\frac{\int_{0}^{R} U W r^{2} d r}{R \int_{0}^{R} U^{2} r d r}
$$

Where $U(\mathrm{~m} / \mathrm{s})$ and $W(\mathrm{~m} / \mathrm{s})$ are the mean axial and tangential velocities at the exit plane of the swirl generator. In this work, we have adopted a swirl number of 0.225 , which is consistent with the swirl number used by Tucker (2004) for his swirl induced jet noise calculations. The Reynolds number is defined in terms of the primary (bulk) axial velocity $(U)$ diameter of the core jet annulus $(D)$ and the kinematic viscosity of air $v$ such that $\operatorname{Re}=U D / v$.

The computational domain considered extended 30 core jet diameters radially and 40 core jet diameters axially with dimensions of approximately $26 m \times 26 m \times 42 m$, for which $140 \times 140 \times 120(2.3$ million $)$ points Cartesian grid aligned along the $\mathrm{x}, \mathrm{y}$ and $\mathrm{z}$ directions respectively was used. The grid lines in the $x$ and $y$ directions used an 
expansion ratio of $\gamma_{x y}=\Delta x(i) / \Delta x(i-1)=1.08$ and an expansion ratio of $\gamma_{z}=1.07$ was used in the z-direction. A grid sensitivity analysis was performed earlier and showed that reasonable grid independence is achieved for the present work.

The inflow mean axial velocity for the core and annular jets, the inflow mean swirl velocity for the annular jet were specified using the power low velocity profile such that

$$
<U>=C_{0} U_{j}\left(1-\frac{|y|}{r}\right)^{1 / 7}
$$

where $U_{j}$ is bulk velocity, $y$ is the radial distance from the jet centre line and $r$ is the radius of the jet. We have used the constant $C_{0}=1.28$, which is consistent with a fully developed turbulent pipe flow outlet. The turbulence fluctuations are generated from a Gaussian random number generator and added to the mean velocity profiles. The method of turbulence generation using a Gaussian random number generator especially for complex swirl flow configurations is consistent and shows good agreement with other inflow generation methods for the swirling flames (Kempf et. al 2008). A free slip boundary condition is used for the side walls and convective outlet boundary condition is applied at the outflow for velocities. For the passive scalar, a top hat profile is specified at the inlet such that for one case, the passive scalar value is 1.0 at core inlet and 0 elsewhere, while for the second case the passive scalar value is 1.0 at annular inlet and 0 elsewhere. For the scalar a zero normal gradient condition is used at outlet. 
The time averaged mean axial and radial velocities, mean passive scalar components and their mean fluctuating values are calculated by time averaging the unsteady variables obtained from LES results, i.e.

$$
<\bar{\phi}>=\frac{1}{N_{t}} \sum_{n=1}^{N_{t}} \bar{\phi}^{n}, \quad \bar{\phi}_{r m s}=\sqrt{\frac{1}{N_{t}} \sum_{n=1}^{N_{t}}\left(\bar{\phi}^{n}-<\bar{\phi}>\right)^{2}}
$$

Where $N_{t}$ represents the number of samples.

The simulations are carried out for ten flow passes transiently and then the statistics collected over another ten flow passes. This allows the flow field to fully develop and initial transients to exit the computational domain. The samples are only taken after the flow field has fully developed.

\section{B. Numerical discretisation}

The program used to perform the simulations is the PUFFIN code developed by Kirkpatrick et al. (2003a, b, 2005) and later extended by Ranga Dinesh (2007). This LES code has been extensively validated for various applications such as turbulent isothermal swirling jets (Malalasekera et al., 2007) and turbulent swirling nonpremixed flames (Malalasekera et al, 2008, Kempf et al. 2008). PUFFIN computes the temporal development of large-scale flow structures by solving the transport equations for the spatially filtered continuity, momentum and passive scalar. The equations are discretised in space with the finite volume formulation using Cartesian coordinates on a non-uniform staggered grid. A second order central differencing scheme (CDS) is used for the spatial discretisation of all terms in both the momentum equation and the pressure correction equation. This minimises the projection error and ensures convergence in conjunction with an iterative solver. The diffusion terms of the passive scalar transport equation are also discretised using the second order CDS. 
The convection term of the scalar transport equation is discretised using the SHARP scheme (Leonard, 1987).

The time derivative of the mixture fraction is approximated using the Crank-Nicolson scheme. The momentum equations are integrated in time using a second order hybrid scheme. Advection terms are calculated explicitly using second order AdamsBashforth, while diffusion terms are calculated implicitly using second order AdamsMoulton to yield an approximate solution for the velocity field. Finally, mass conservation is enforced through a pressure correction step.

The time step is varied to ensure that the Courant number $C_{o}=\Delta t u_{i} / \Delta x_{i}$ remains approximately constant where $\Delta x_{i}$ is the cell width, $\Delta t$ is the time step and $u_{i}$ is the velocity component in the $x_{i}$ direction. The solution is advanced with a time stepping corresponding to a Courant number in the range of $C_{o}=0.3$ to 0.5. The Bi-Conjugate Gradient Stabilized (BiCGStab) method with a Modified Strongly Implicit (MSI) preconditioner is used to solve the system of algebraic equations resulting from the discretisation.

\section{RESULTS AND DISCUSSION}

This section discusses the results obtained from the LES calculations. First we discuss the velocity fields and then describe the passive scalar fields for two cases considered. Both sections discuss the velocity and passive scalar fields based on instantaneous and time averaged quantities for different axial locations. The radial plots of the mean and rms quantities have been normalised by the diameter of the annular jet $\left(D_{a}\right)$. 


\section{Analysis of the results for velocity field}

Contour plots for the mean axial and swirl velocities are shown in Figure 1 a,b. The centreline mean axial velocity is only minimally influenced by the annular swirl in the near field, $(0<x / D<5.0)$, but the contour plot for the mean swirling velocity (Figure 1, b) displays an overall axi-symmetric distribution of the mean swirl velocity with the largest values found close to the annular inlet and then decaying further downstream. The swirl velocity results in a higher spreading rate for the flow field and faster decay for the mean axial velocity in the near field as observed by other investigators in their LES calculations for a coaxial swirl jet in unconfined environment (Garcia-Villalba et al., 2006, Frohlich et al., 2008).

Figure $2(a, b)$ show the velocity vector field obtained from the time averaged mean velocities, which shows the development of the mean velocity profiles and its expansion in the radial direction within the intermediate and far field regions. However, a typical swirl induced recirculation zone (Gupta et al. 1984) does not appear, with absence of low pressure regions in the flow field, due to the existing low swirl number we used of 0.225 . The vector field also shows that the jet has an outward radial component due to the centrifugal forces which widens considerably at further downstream locations.

Figure 3 shows contours of instantaneous passive scalar levels and indicates two shear layers produced for the simulated coaxial swirl jet with the inner layer forming between the core jet and the annular jet and the outer layer established at the border between the annular region and surrounding flow. The shear layers contain curvatures as a result of swirl and the presence of swirl produces a pressure drop in the 
momentum transport equations and increases the centrifugal force close to the annular region (Gupta et al. 1984). The addition of swirl also increases the turbulence and thus increases the velocity fluctuations especially in the inner mixing region. The vortices start to roll up at a close distance to the inlets due to the centrifugal force and the rate of spreading increases sharply between the shear layers which significantly enhance the mixing. The existence of small eddies and their roll-up also increases the intermittency in both velocity and scalar fields.

The radial plots of the time averaged mean axial velocity are shown in Figure 4. The profiles extended from $x / D=0.5$ to $x / D=30.0$ and the plots show that the mean axial velocity follows the axi-symmetric behaviour. With the additions of swirl, radial spread of the mean axial velocity increases in intermediate regions $(x / D=3.0,5.0)$ and possesses a flat region at far field axial locations. The existence of the swirl velocity also reduces the higher axial momentum generated from the inlet axial velocities and hence increases the spread rate of the axial velocity towards radial direction. As reported in literature a weak coflow is usually introduced in the simulations of turbulent free jets (Stanley et al. 2002) and hence has no significant effect for the jet dynamics. However, higher coannular jet velocity does affect the central jet momentum seen in the present case.

Figure 5 shows the time averaged mean radial velocity at $\mathrm{x} / \mathrm{D}=0.5,1.0,2.0,3.0,5.0$, 10.0, 20.0, 30.0. The mean radial velocity is close to zero near the centreline at all axial locations and sharply reduces to negative values above the annular jet due to the swirl velocity. A sudden reduction of the mean radial velocity is also visible at near field axial locations such as $\mathrm{x} / \mathrm{D}=0.5,1.0,2.0$. The reduction of the mean radial 
velocity over the annular jet is smooth for intermediate region and possesses a flat shape at far field axial locations. Time averaged mean swirling velocity is shown in Figure 6. Since the swirl velocity component is introduced from the annular jet with swirl number 0.225 , the radial profiles of the mean swirl velocity has higher values above the annular inlet. These higher swirling velocity values has extend up to $\mathrm{x} / \mathrm{D}=10$ and then gradually decrease at far field locations.

Figures 7-9 show the root mean square (rms) fluctuations of axial, radial and swirling velocities. The highest peak value of the rms axial fluctuation appears at $\mathrm{r} / \mathrm{D}=-0.5,0.5$ (Figure 7) which is a direct consequence of the vortex shedding in the inner mixing region between inner potential core and outer potential core. Further downstream the axial fluctuation follows the same profile shape as mean axial velocity. As seen in Figure 8 , the rms radial fluctuation has a small peak in region above the annular jet and eventually increases the peak value at intermediate regions $(\mathrm{x} / \mathrm{D}=2.0,3.0,5.0)$. The maximum peak values are significantly lower than those for the rms axial fluctuations, as also observed by Ribeiro and Whitelaw (1980) for their coaxial swirl experiment. The centreline fluctuations increase in the far field and follow the same distribution shape as the rms axial velocity at $\mathrm{x} / \mathrm{D}=10.0,20.0,30.0$ while axial and radial fluctuations values are much the same at downstream axial locations. The rms swirling fluctuations (Figure 9) also follow the same profile shape as radial fluctuations, but the peak values are higher than rms radial fluctuations and lower than the rms axial fluctuations at near field axial locations.

The overall agreement for the time averaged velocity components between the present work and previous experimental studies undertaken by Gupta et al. (1984), Bender 
and Buchner (2005), and previous numerical studies carried out by McIlwain and Pollard (2002), Garcia-Villalba et al. (2006) is found to be consistent. Thus the present results suggest that the LES technique can provide reliable details for the coaxial swirl jet development. However since turbulent free shear flows display an intermittent behaviour, future analysis on intermittency of intermittency on both velocity and passive scalar would seems to be useful in the presence of swirl.

\section{Analysis of the results for scalar field}

The passive scalar distributions have been studied for two further cases. Case 1 simulated the scalar distribution by setting the scalar value to 1 for the inlet core and zero elsewhere, while case 2 simulated the scalar distribution by specifying a value of 1 for the inlet annular jet and zero elsewhere. Both simulations have been done independently and statistics collected for the time averaged calculations.

Figure 10 and 11 show the snapshots and filtered passive scalars for case 1 and case 2 respectively. Since the swirl velocity was introduced via the annular jet, the swirl effect on core is not significant and produces less mixing in near field region for case 1. Furthermore the axial velocity ratio between the core and annular jets is high (1.48) and hence the dominant higher core jet velocity takes the passive scalar further down with less mixing for case 1 . The swirl velocity gradually increases its effect on core jet within the intermediate region which helps to form different vortex patterns and to spread the scalar value radially at downstream axial locations. However, the mixing is different for case 2 in which, the swirl velocity is introduced via the annular jet where the passive scalar value is now initialised as 1 at inlet. As seen in Figure 11, the passive scalar for case 2 shows more mixing within the intermediate region due to the 
direct impact of the swirl velocity. The swirl induced vortical structures directly help to enhance the mixing at this stage of development. The different effect of swirl on the passive scalar mixing for both cases is confirmed by the radial plots of the time averaged mean passive scalars at different axial locations (Figure 12).

The mean passive scalar follows the Gaussian shape in both near and far fields. The radial profiles for case 1 show less mixing close to centreline at the more upstream locations $(\mathrm{x} / \mathrm{D}=0.5,1.0,2.0,3.0,5.0)$. The radial spread of the passive scalar starts to expand more at downstream positions. In case 2, the scalar values distribute more towards the radial direction with a reduced maximum value in intermediate regions due to the direct effect of the swirl velocity. However, the swirl velocity has a greater effect on mixing for case 1 than case 2 in the far field and hence absolute scalar

values for case 1 reduce more than values for case 2 on the plots ( $\mathrm{x} / \mathrm{D}=20.0,30.0)$. This analysis shows that the spreading rate of the passive scalar changes with respect to swirl strength and its local velocity value in each case. Significant intermittency in both velocity and passive scalar fields can be expected and hence investigations of probability density functions and radial spread of the intermittency of both velocity and passive scalar be useful to explain the changes occurring in flow mixing in the two cases considered.

\section{Analysis of the results for intermittency}

The intermittency is another important physical issue that could influence with the mixing process. Turbulent free shear flows with free boundaries as considered in the present work display an intermittent behaviour especially close to the outer edge of the flow where the flow alternates between rotational and irrotational states. 
Mathematically, intermittency can be classified using an indicator function with the value of one in turbulent regions and zero in non-turbulent regions. It represents the fraction of a time interval during which a point is inside the turbulent fluid. Here we consider the derivation of intermittency for both velocity and passive scalar fields for the coaxial swirl jet. A variety of methods exist for determining the intermittency of a flow. A normalised histogram method experimentally tested by Andreotti (1999) is commonly used to estimate the probability density function (PDF). The intermittency value can be calculated using a summation of probability values with a threshold for given instantaneous values. Here we assume that the PDF is smooth at the scale of one histogram bin and calculate PDF's similar to the method used by Schefer and Dibble (2001). We consider more than 8000 measurements and at each selected spatial location are 50 bins over the $3-\sigma$ limits of the data. The PDF's are normalised and thus

$$
\int_{0}^{1} P(f) d f=1
$$

Therefore the intermittency value $\gamma$ can be obtained from the probability values in the presence of a threshold value such that

$$
\gamma=P\left(f>f_{t h}\right)
$$

Here we selected a threshold value of $13.1 \mathrm{~m} / \mathrm{s}$ for the velocity which is equal to $\left(U_{c}+U_{a}\right) / 10$ and a threshold value of 0.015 for the passive scalar which is similar to the value considered by Schefer and Dibble (2001) for their intermittency of an isolated round jet. Figures 13 and 14 shows the probability density distributions (PDF) of the instantaneous axial velocity and passive scalar at $\mathrm{x} / \mathrm{D}=30$. The PDF's of the velocity exhibit Gaussian shape close to the centreline, Figure 13(a,b). However, 
the PDF shape is shifting to non-Gaussianity with increase radial distance and thus indicates high rotational to irrotational variations towards the outer edge of the flow. This feature can be further studied using the radial intermittency plots. The pdfs of passive scalar also follow the same behaviour and shows a Gaussian shape intermittency in Figure $14(a, b)$ and then non-Gaussian at far field radial locations. The Gaussian like distributions are relatively wider near the centreline (Figure 13,14 $(a, b))$ and getting smaller with the increase radial distance (Figure 13,14 (c,d)) for both velocity and passive scalar fields.

Figure 15 shows the radial variation of velocity intermittency at $x / D=10,20$ and 30 . The velocity intermittency value is unity in the centreline region at $\mathrm{x} / \mathrm{D}=10,20$, but suddenly start to drop down at $\mathrm{x} / \mathrm{D}=30$ in the region close to the centreline. The value of unity indicates a fully turbulent situation on the centreline. The velocity intermittency plot at $\mathrm{x} / \mathrm{D}=10$ indicates only a low level intermittent behaviour and eventually a change to laminar flow with increased radial distance. The intermittent behaviour of the velocity increased at $x / D=20,30$ and shifts to a non-Gaussian shape at $\mathrm{x} / \mathrm{D}=30$. The addition of swirl from the annular stream creates more complex flow patterns in the instantaneous flow field and thus more highly intermittent behaviour for the coaxial swirl jet. The radial variation of the passive scalar intermittency is shown in Figure 16. It can be seen that the radial spread of the passive scalar intermittency is wider than that observed for the velocity field. Moving from the centreline into a region with $0<\gamma<1$ an unmixed surrounding medium is observed. The intermittency profiles for velocity and passive scalar are similar at near field $(\mathrm{x} / \mathrm{D}=10)$ and differences are wider at $\mathrm{x} / \mathrm{D}=20$ and 30 . The intermittency of passive 
scalar shows more rotational/irrotational variations in the region $0.5<\mathrm{r} / \mathrm{D}<4$ at $\mathrm{x} / \mathrm{D}=20$ and 30. The profiles also follow the Gaussian shape at all three axial locations.

\section{CONCLUSION}

A turbulent swirling coaxial swirl jet with high inlet axial velocities has been simulated using LES. Swirl has been introduced into the initial annular jet with a swirl number of 0.225. A passive scalar was introduced into the core and annular jet regions separately. The simulations produced both instantaneous and time averaged quantities to describe the flow and mixing fields. Radial plots of mean and rms of velocity field have been discussed along with the corresponding passive scalar distributions. The mean and fluctuation fields captured the potential cores and higher turbulence levels inside the inner mixing regions due to vortex shedding. The time averaged mean scalar distributions demonstrated the impact of the swirl on coaxial jet mixing at near field and far field locations. The calculated mean velocities, rms fluctuations and passive scalar results suggest that the coaxial swirl jet exhibits intermittent behaviour in some regions of the flow and the corresponding intermittency profiled have been obtained for both velocity and scalar fields. Derived probability density functions for both velocity and passive scalar indicate complex mixing and radial intermittency plots reveal sudden changes between rotational and irrotational in the regions outer edge of the flow domain. The rapid mixing process also benefited by the swirl velocity component introduced from the annular inlet. 


\section{ACKNOWLEDGEMENT}

We are grateful to the EPSRC for their financial support under grant EP/E036945/1 on the Modelling and Simulation of Intermittent Flows. 


\section{REFERENCES}

Akselvoll, K, Moin, P. (1996) 'Large eddy simulation of turbulent confined coannular jets', J. Fluid Mechanics, 315, 387-411

Andreotti, B, Douady, S. (1999) 'On probability distribution functions in turbulence. Part 1. A regularisation method to improve the estimate of a PDF from an experimental histogram', Physica D 132, 111-132

Apte, S.V, Mahesh, K, Moin, P, Oefelein, J.C. (2003) 'Large eddy simulation of swirling particle laden flows in a coaxial jet combustor', Int J. Multiphase Flows 29(8), 1311-1331

Bender, C, Buchner, H. (2005) 'Noise emissions from a premixed swirl combustor', In Proc. $12^{\text {th }}$ Int. Congress on Sound and Vibration, Lisbon, Portugal

Billant, P, Chomaz, J.M, Huerre, P. (1998) 'Experimental study of vortex breakdown in swirling jets', J. Fluid Mech., 376, 183-219

Byggstoyl, S, Kollmann, W. (1981) 'Closure model for intermittent turbulent flows', Int. J. Heat and Mass Transfer', 24(11), 1811-1822

Bear, J.M. and Chigier, N.A. (1972) 'Combustion Aerodynamics', Wiley, New-York, NY 
Champagne, F.H, Kromat, S. (2000) 'Experiments on the formation of the recirculation zone in swirling coaxial jets', Exp. Therm. Fluid Sci., 29, 494-504

Cho, J.R, Chung, M.K. (1992) 'A $k-\varepsilon-\gamma$ equation turbulence model', J. Fluid Mech.,' 237, 301-322

Dianat, M, Yang, Z, Jiang, D, McGuirk, J.J. (2006) 'Large eddy simulation of scalar mixing in a coaxial confined jet', Flow Turb. Combust., 77, 205-227

Garcia-Villalba, Frohlich, J, Rodi, W. (2006) 'Identification and analysis of coherent structures in the near field of a turbulent confined annular swirling jet using large eddy simulation', Phy. of Fluids, 18, 1-17

Garnier, F., Baudoin, C., Woods, P. and Louisnard, N. (1997) 'Engine emissions alteration in the near field of an aircraft', J. Atmos. Environ. 31, pp.1767-1781

Giannadakis, A, Perrakis, K, Panidis, T.H. (2008) 'A swirling jet under the influence of a coaxial flow', Exp. Therm. Fluid Sci., 32(8), 1548-1563

Gupta, A.K, Lilly, D.G, Syred, N. (1984), Swirl Flows, Abacus, Kent, England

Guo, B., Langrish, T.A.G., Fletcher, D.F. (2001) 'Simulation of turbulent swirl flow in an axisymmetric sudden expansion', AIAA J. 39, 96-102 
Gouldin, F.C, Depsky, J.S, Lee, S.L. (1985) 'Velocity field characteristics of a swirl flow combustor', AIAA J., 23, 95-102

Kollomann, W, Janicka, J. (1982) 'The probability function of a passive scalar in turbulent shear flows', Phy. Fluids, 25(10), 1755-1769

Kirkpatrick, M.P., Armfield, S.W. and Kent J.H. (2003a) 'A representation of curved boundaries for the solution of the Navier-Stokes equations on a staggered threedimensional Cartesian grid', J. of Comput. Physics ,184, 1-36.

Kirkpatrick, M.P., Armfield, S.W., Masri, A.R. Ibrahim, S.S. (2003b) 'Large eddy simulation of a propagating turbulent premixed flame', Flow Turb. Combust., 70 (1) $1-19$.

Kirkpatrick, M.P. and Armfield, S.W. (2005) 'Experimental and large eddy simulation results for the purging of a salt water filled cavity by an overflow of fresh water', Int. J. of Heat and Mass Transfer, 48 (2), 341-359

Kempf, A, Malalasekera, W, Ranga Dinesh, K.K.J, Stein, O. (2008) 'Large eddy simulation with swirling non-premixed flames with flamelet model: A comparison of numerical methods', Flow Turb. Combust.,81, 523-561

Lu, X, Wang, S, Sung, H, Hsieh, A, Yang, V. (2005) 'Large eddy simulations of turbulent swirling flows injected into a dump chamber', J. Fluid Mech., 572, 171-195 
Leonard, B.P. (1987) 'SHARP simulation of discontinuities in highly convective steady flows', NASA Tech. Memo., 100240

McIlwain, S, Pollard, A. (2002) 'Large eddy simulation of the effects of mild swirl on the near field of a round free jet', Phy. of Fluids, 14 (2), 653-661

Malalasekera, W, Ranga Dinesh, K.K.J, Ibrahim, S.S., Kirkpatrick, M.P. (2007) 'Large eddy simulation of isothermal turbulent swirling jets', Combut. Sci. Tech. 179, pp. 1481-1525.

Malalsekera,W, Ranga Dinesh, K.K.J, Ibrahim, S.S, Masri, A.R. (2008) 'LES of recirculation and vortex breakdown in swirling flames', Combust. Sci. Tech. 180, $809-832$

Mattingly, J.D, Oates, G.C. (1986) 'An experimental investigation of the mixing of coannular swirling flow, AIAA J., 24, 785-792

Pierce, C, Moin, P. (1998) 'Large eddy simulation of a confined coaxial jet with swirl and heat release', AIAA Paper no 98-2892

Piomelli, U. and Liu, J. (1995) 'Large eddy simulation of channel flows using a localized dynamic model', Phy. Fluids, 7, 839-848

Ranga Dinesh K.K.J. (2007) 'Large eddy simulation of turbulent swirling flames', PhD Thesis, Loughborough University, UK 
Ribeiro, M.M, Whitelaw, J.H. (1980) 'Coaxial jet with and without swirl', J. Fluid Mech., 96, 769-795

Roback, R, Johnson, B.V. (1983) 'Mass and momentum turbulent transport experiments with confined swirling coaxial jets', NASA CR-168252

Schefer, R.W, Dibble, R.W. (2001) 'Mixture fraction field in a turbulent nonreacting propane jet', AIAA J. 39, 64-72

Smagorinsky, J. (1963) 'General circulation experiments with the primitive equations', M. Weather Review, 91, 99-164

Stanley, S.A, Sarkar, S, Mellado, J.P. (2002) 'A study of the flow field evolution and mixing in a planer turbulent jet using direct numerical simulation', J. of Fluid Mech., $450,377-407$

Tucker, P. (2004) 'Novel MILES computations for jet flows and noise', Int. J. Heat and Fluid Flow, 25, 625-635

Wang.P and Bai, X,S, Wessman, M and Klingmann, J. (2004) 'Large eddy simulation and experimental studies of a confined turbulent swirling flow', Phy. Fluids 16, 33063324 
Wicker, R.B, Eaton, J.K. (2001) 'Structure of a swirling recirculating coaxial free jets and its effect on particle motion, Int. J. Multi. Flow, 27, 949-970

Wegner, B, Staufer, M, Sadiki, A, Janicka, J. (2007) 'Study of flow and mixing in a generic GT combustor using LES', Flow Turb. Combust., 79 (4), 389-403

Wang, S, Yang, V, Hsiao, G, Hsieh, S, Mongia, H.C. (2007) 'Large eddy simulation of gas turbine swirl injector flow dynamics', J. Fluid Mech. 583, 99-122 


\section{FIGURE CAPTIONS}

Figure 1. Contour plot of the time averaged mean axial velocity (a) and mean swirl velocity (b)

Figure 2. Velocity vector field generated from mean velocities (a) actual domain (b) zoom view

Figure 3. Shear layers captured from the LES calculation: contours of instantaneous passive scalar and contour levels are 0.05 to 0.9

Figure 4. Radial plots of time averaged mean axial velocity at different axial locations

Figure 5. Radial plots of time averaged mean radial velocity at different axial locations

Figure 6. Radial plots of time averaged mean swirling velocity at different axial locations

Figure 7. Radial plots of time averaged rms axial velocity at different axial locations

Figure 8. Radial plots of time averaged rms radial velocity at different axial locations 
Figure 9. Radial plots of time averaged rms swirling velocity at different axial locations

Figure 10. Snapshot of the passive scalar mixing for the case 1

Figure 11. Snapshot of the passive scalar mixing for the case 2

Figure 12. Radial plots of time averaged mean passive scalar at different axial locations, solid line indicates case 1 results and dashed line represents case 2 results

Figure 13. Velocity probability density functions (pdf) at $\mathrm{x} / \mathrm{D}=30$

Figure 14. Passive scalar probability density functions (pdf) at $x / D=30$

Figure 15. Radial variation of velocity intermittency at (a) $x / D=10$ (b) $x / D=20$ and (c) $x / D=30$

Figure 16. Radial variation of scalar intermittency at (a) $x / D=10$ (b) $x / D=20$ and (c) $\mathrm{x} / \mathrm{D}=30$ 


\section{FIGURES}
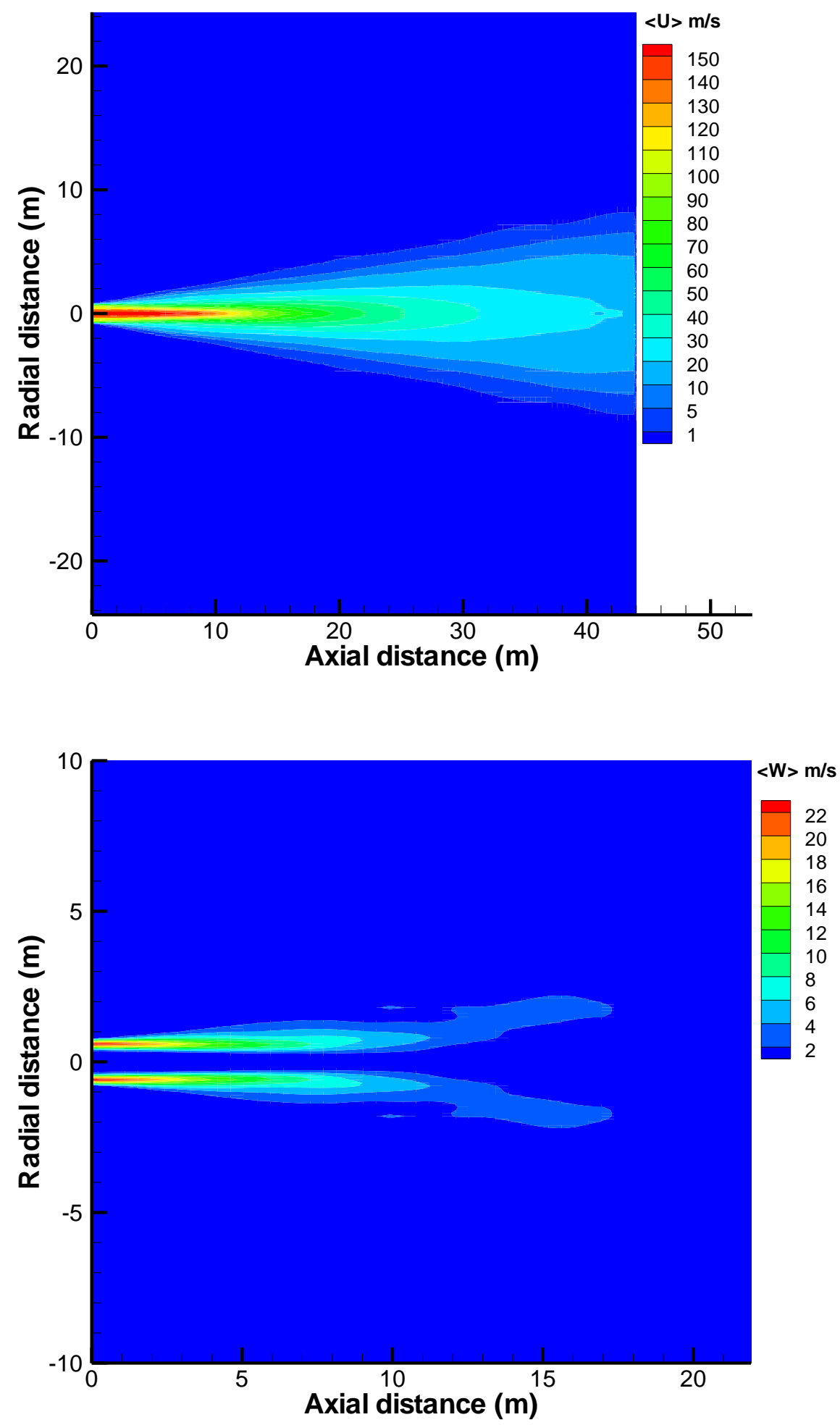

Figure 1. Contour plot of the time averaged mean axial velocity (a) and mean swirl velocity (b) 

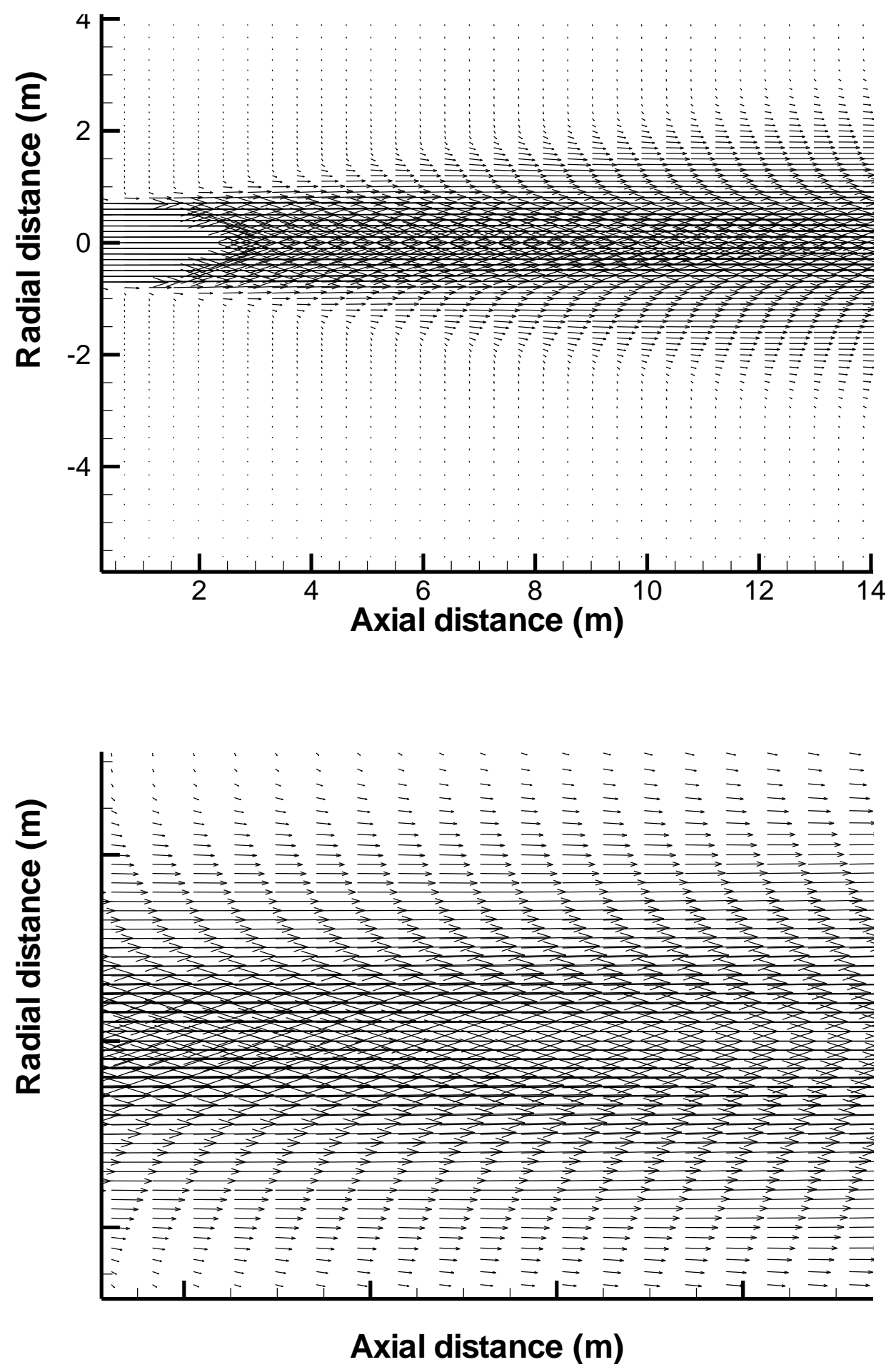

Figure 2. Velocity vector field generated from mean velocities (a) actual domain (b) zoom view 


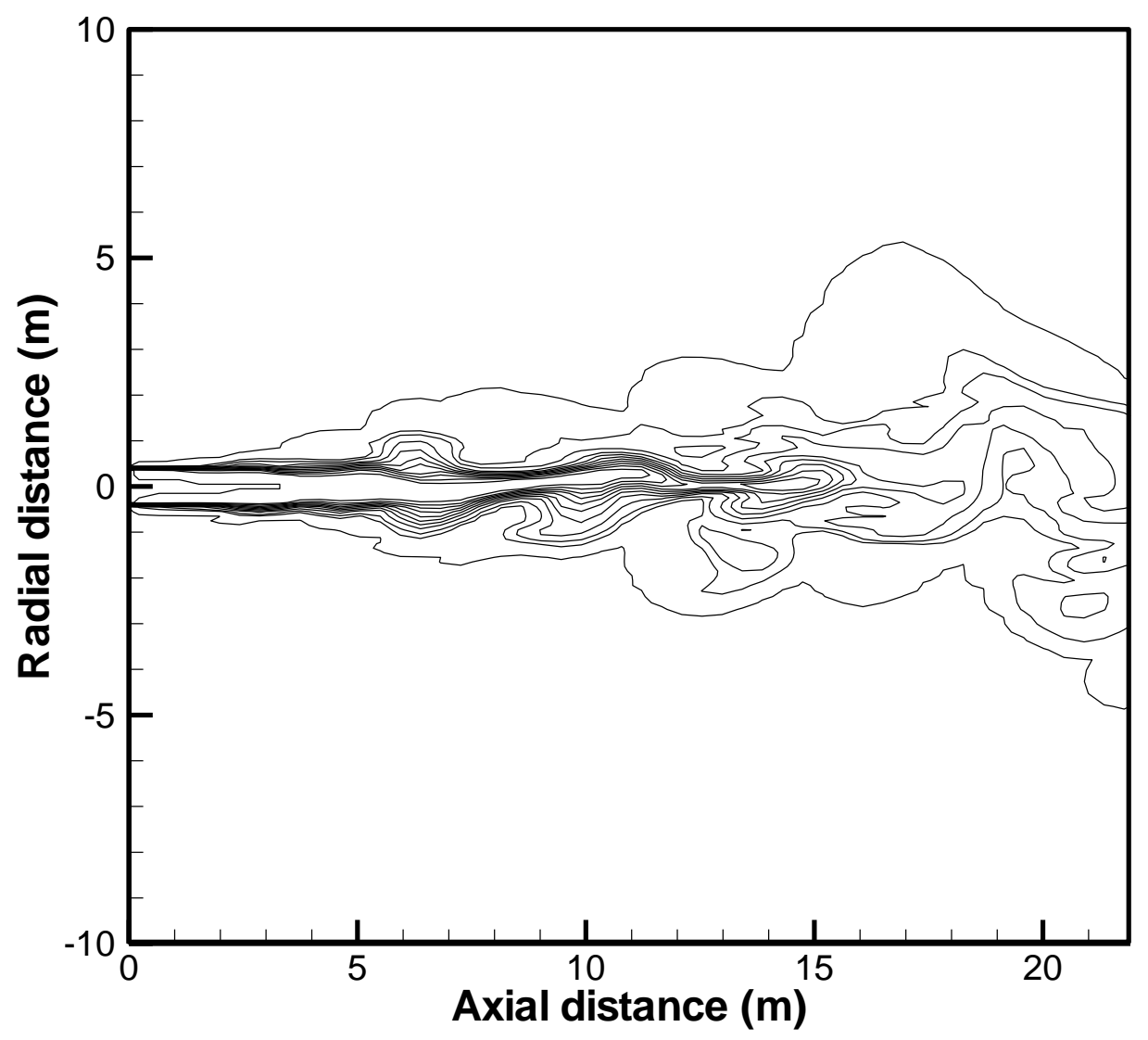

Figure 3. Shear layers captured from the LES calculation: contours of instantaneous passive scalar and contour levels are 0.05 to 0.9 

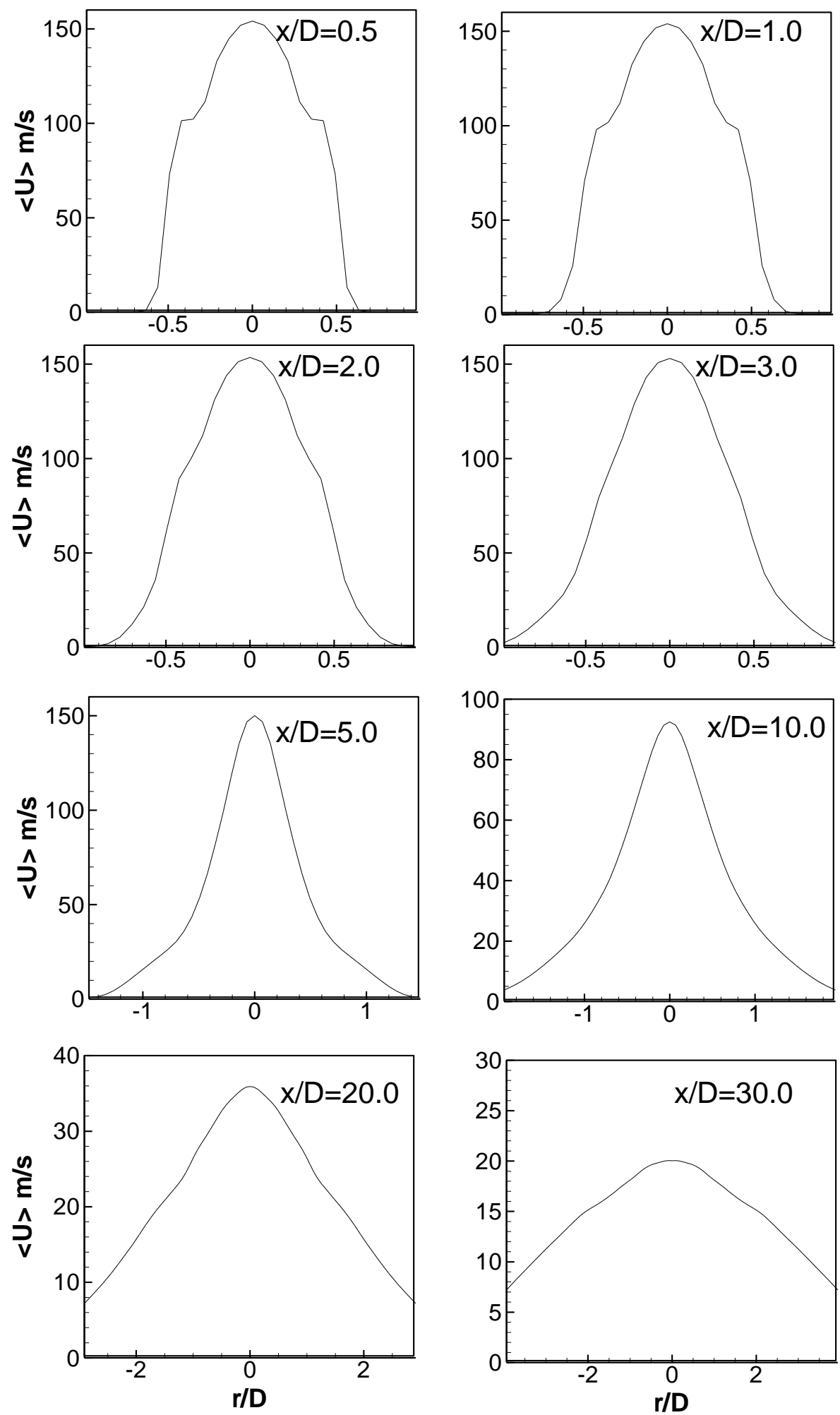

Figure 4. Radial plots of time averaged mean axial velocity at different axial locations 

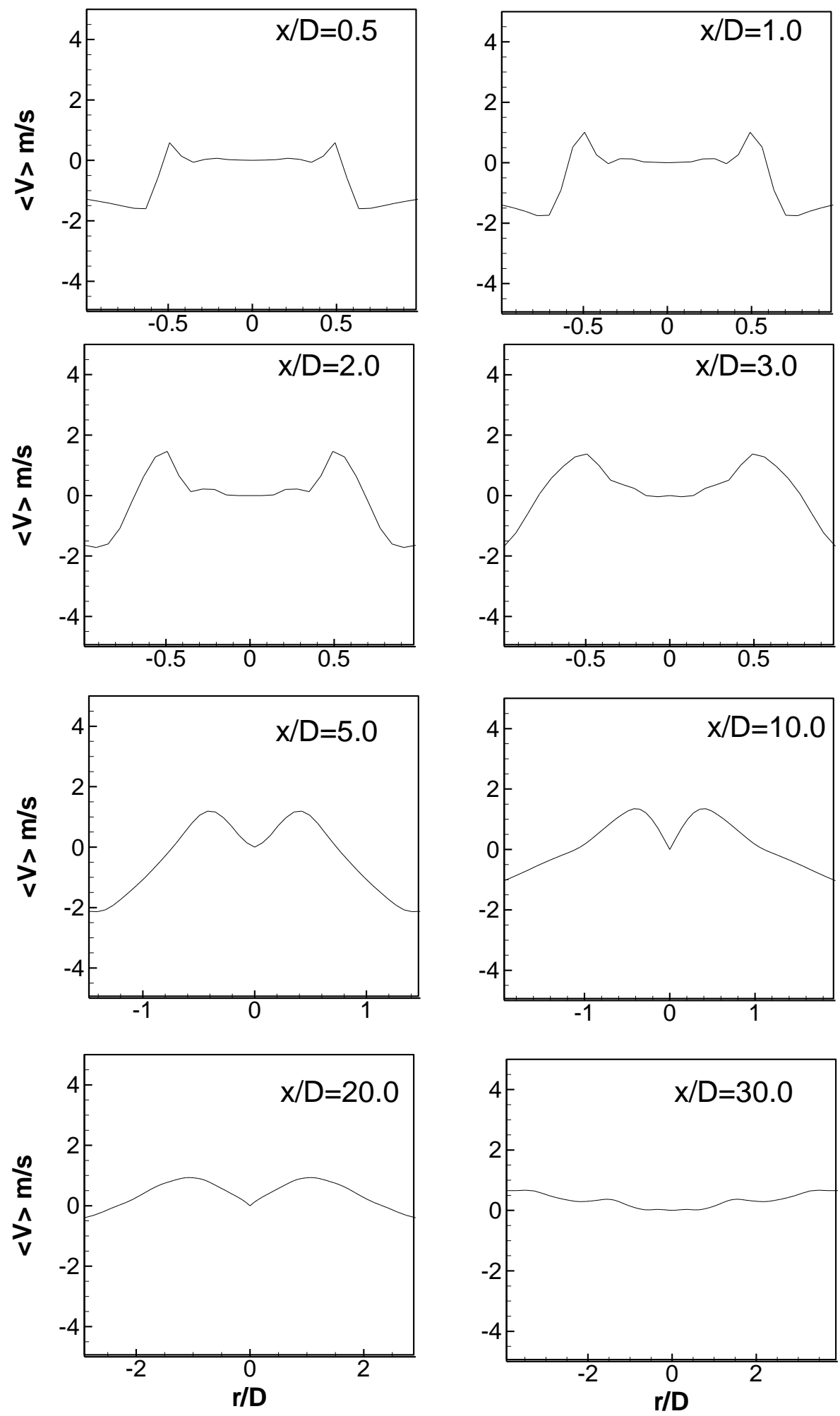

Figure 5. Radial plots of time averaged mean radial velocity at different axial locations 

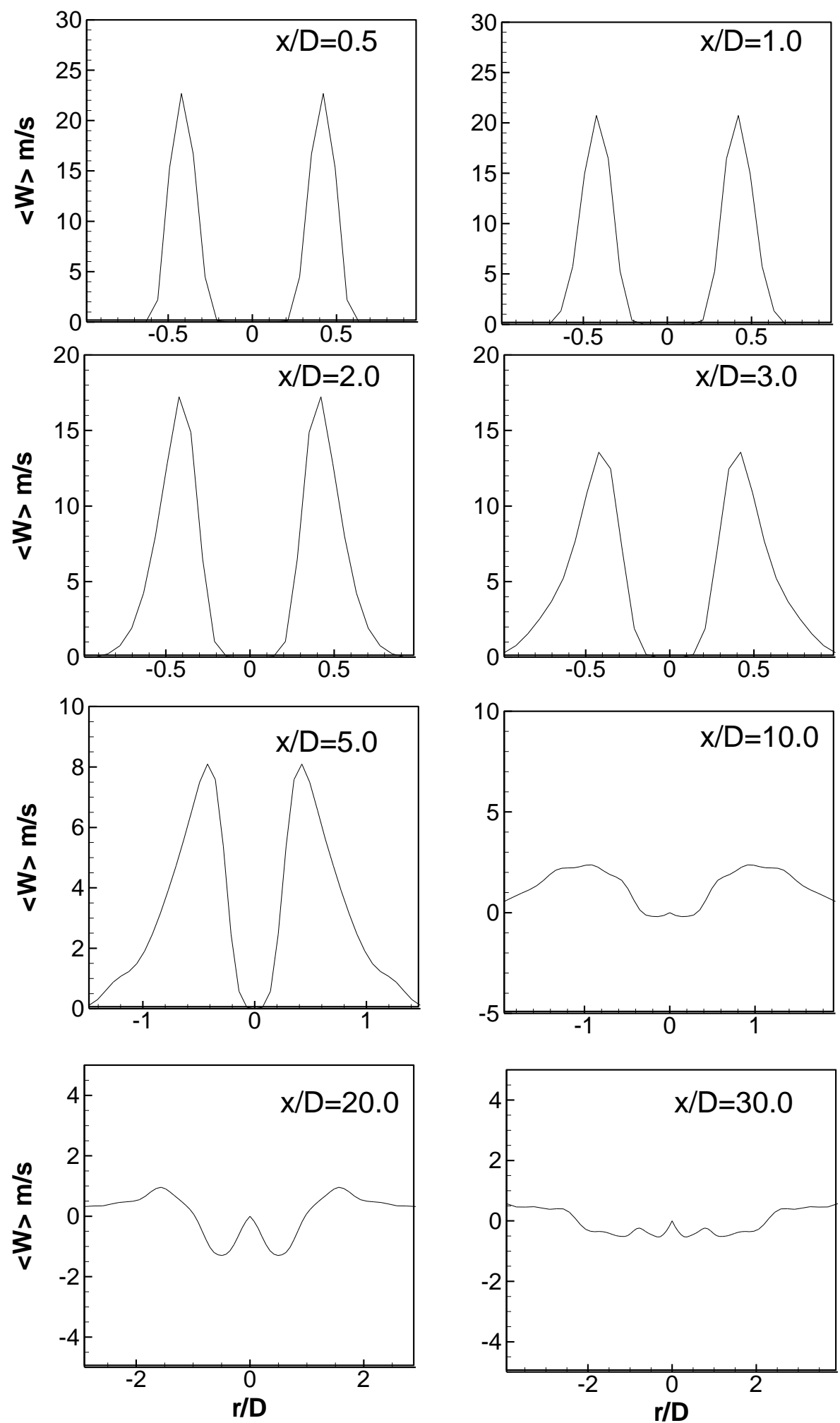

Figure 6. Radial plots of time averaged mean swirling velocity at different axial locations 

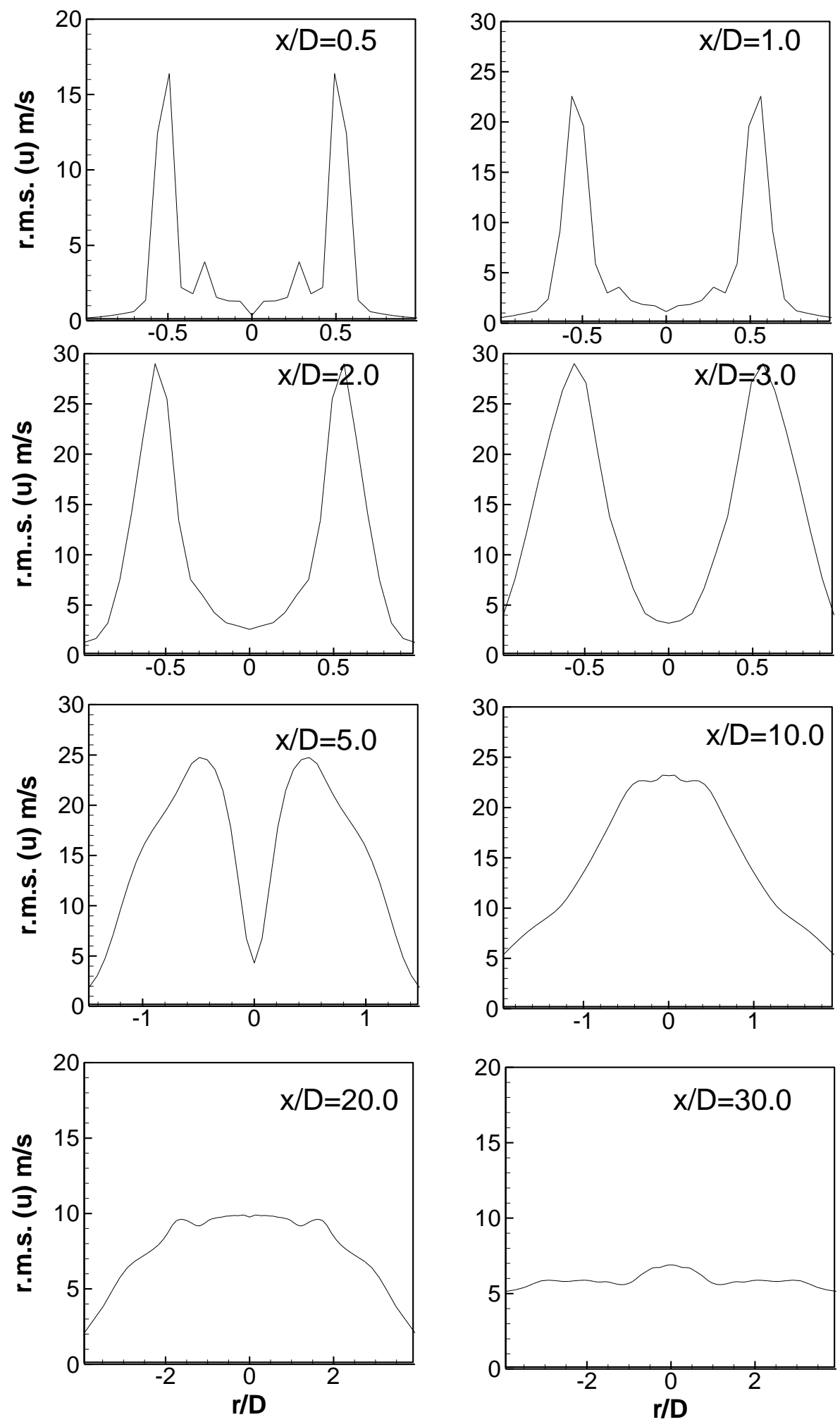

Figure 7. Radial plots of time averaged rms axial velocity at different axial locations 

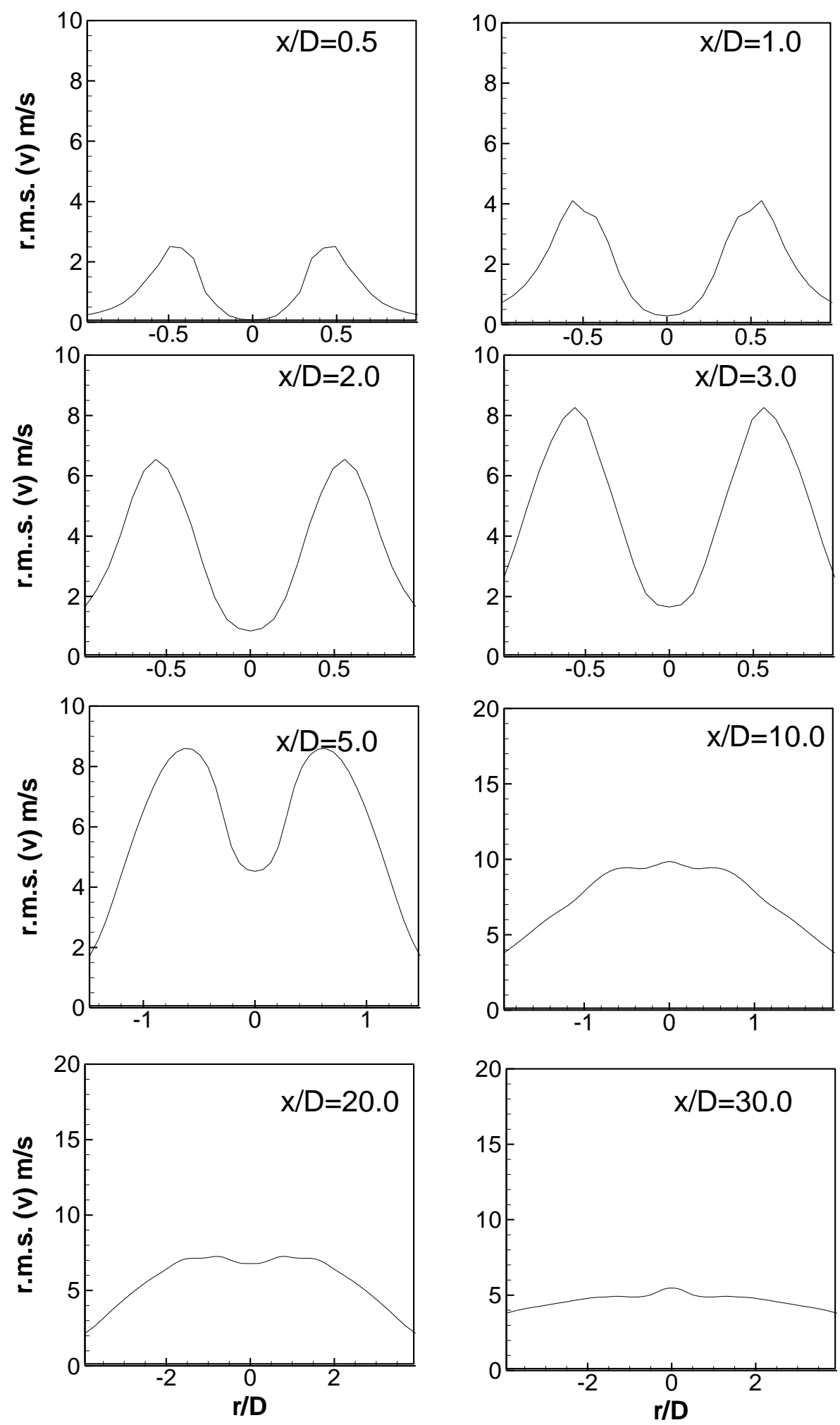

Figure 8. Radial plots of time averaged rms radial velocity at different axial locations 

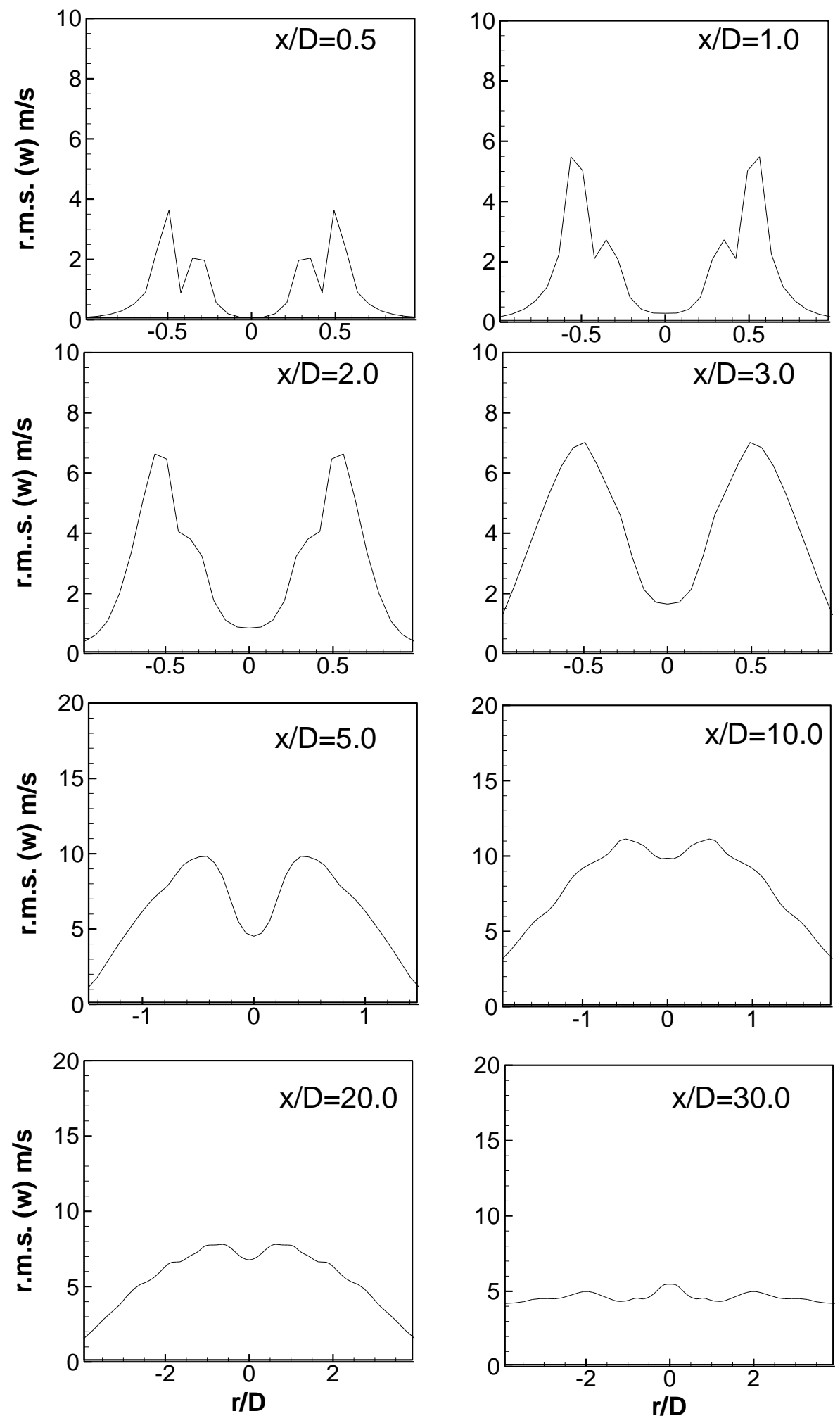

Figure 9. Radial plots of time averaged rms swirling velocity at different axial locations 


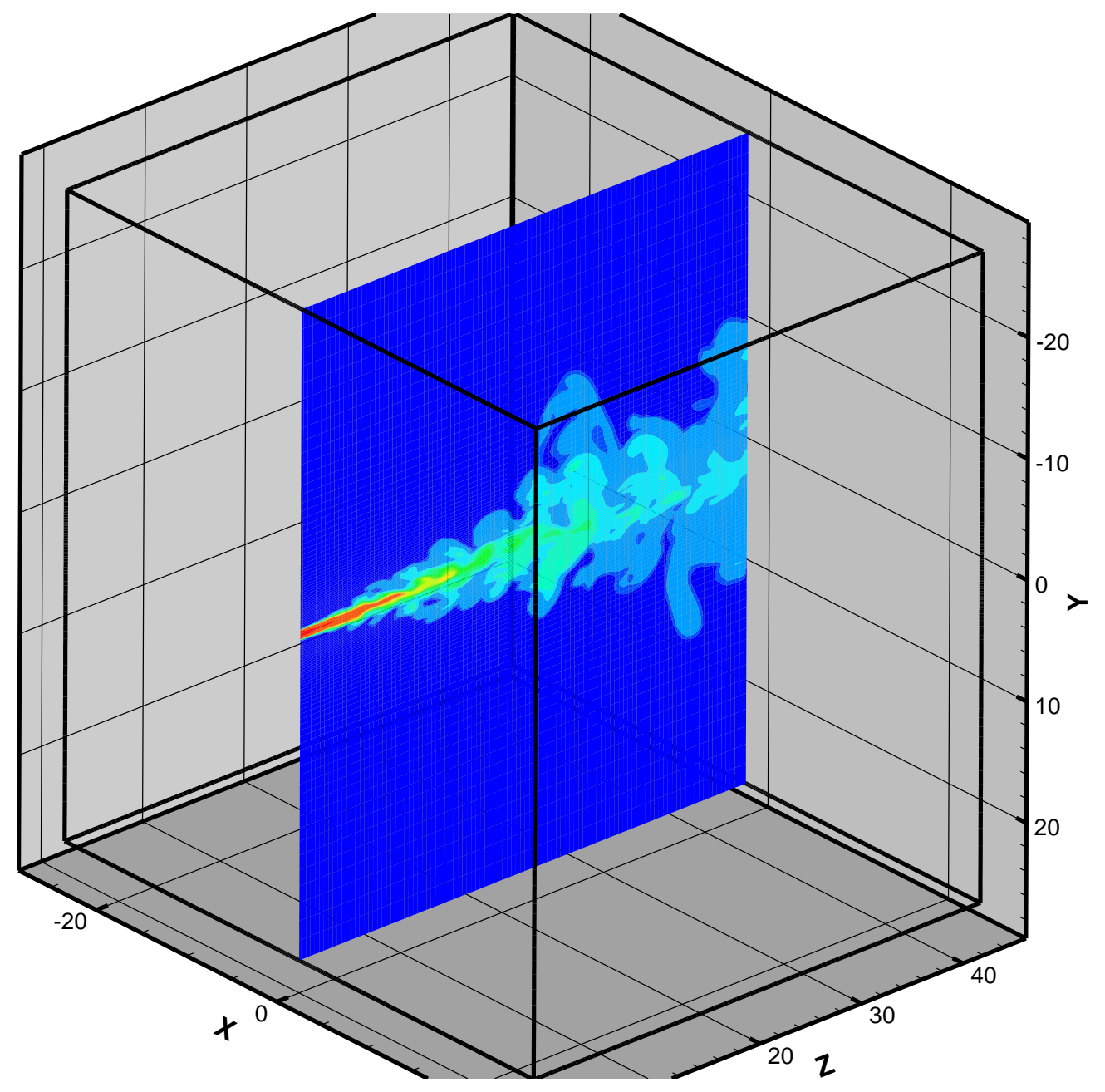

Figure 10. Snapshot of the passive scalar mixing for the case 1 


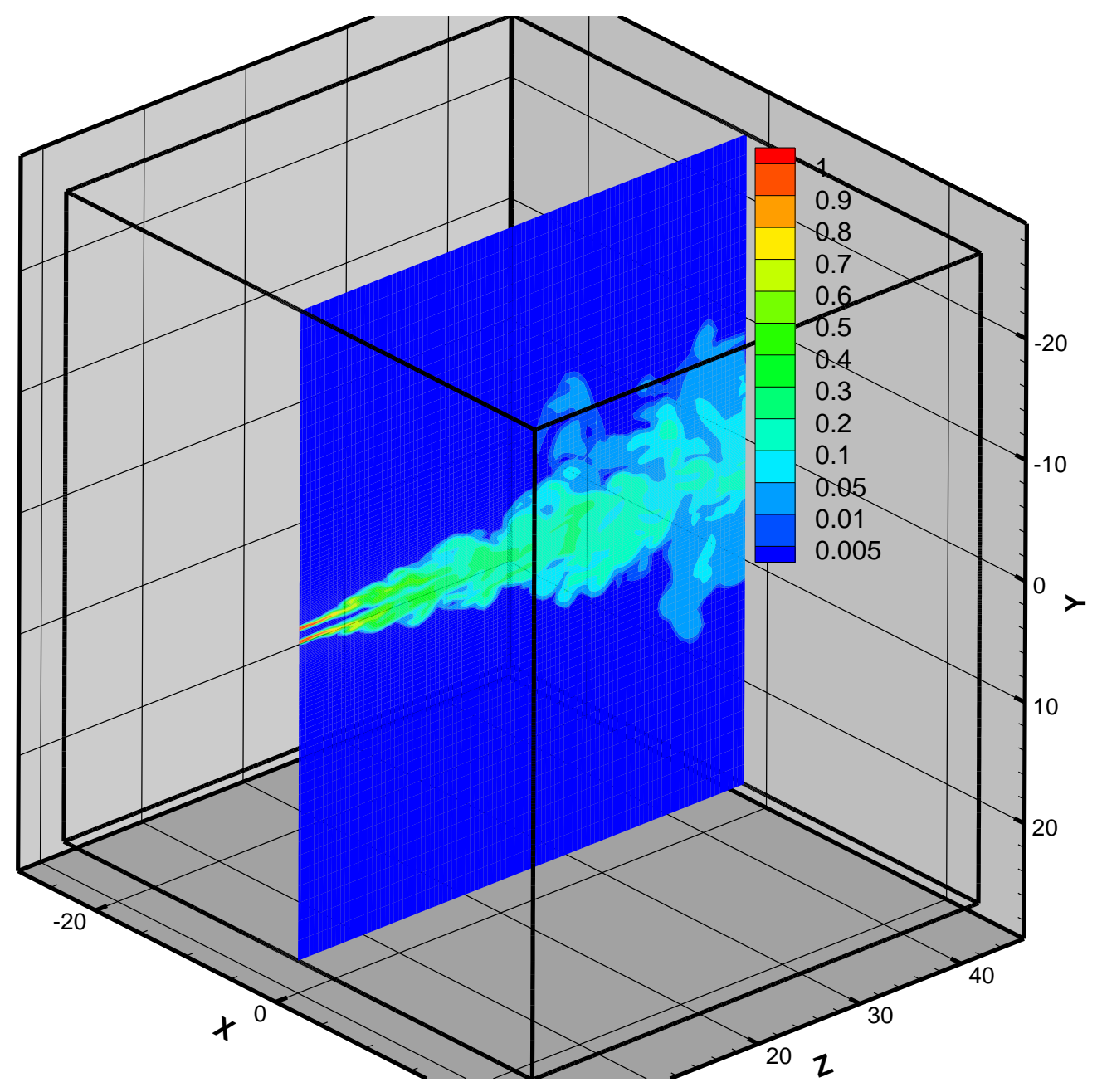

Figure 11. Snapshot of the passive scalar mixing for the case 2 

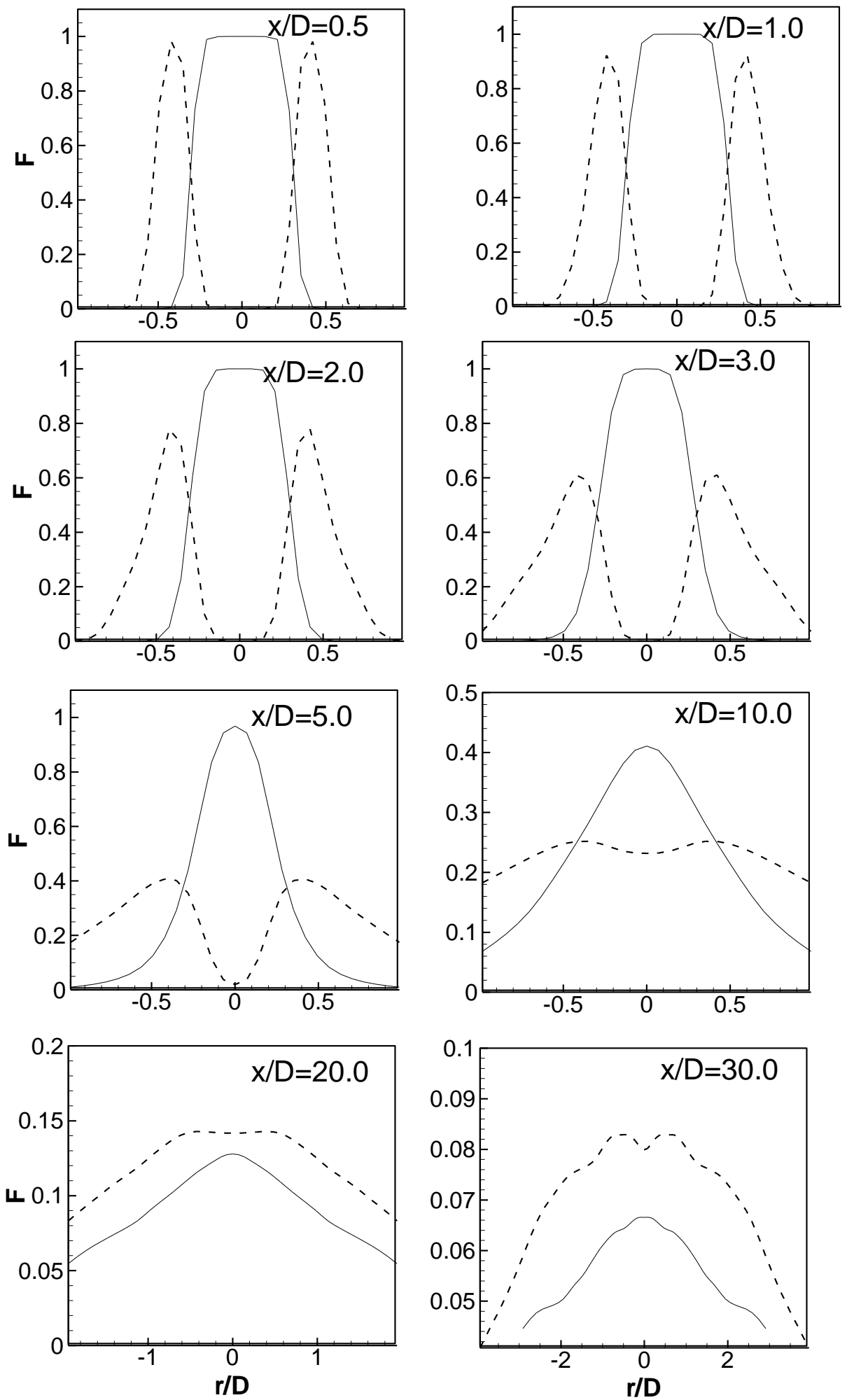

Figure 12. Radial plots of time averaged mean passive scalar at different axial locations, solid line indicates case 1 results and dashed line represents case 2 results 

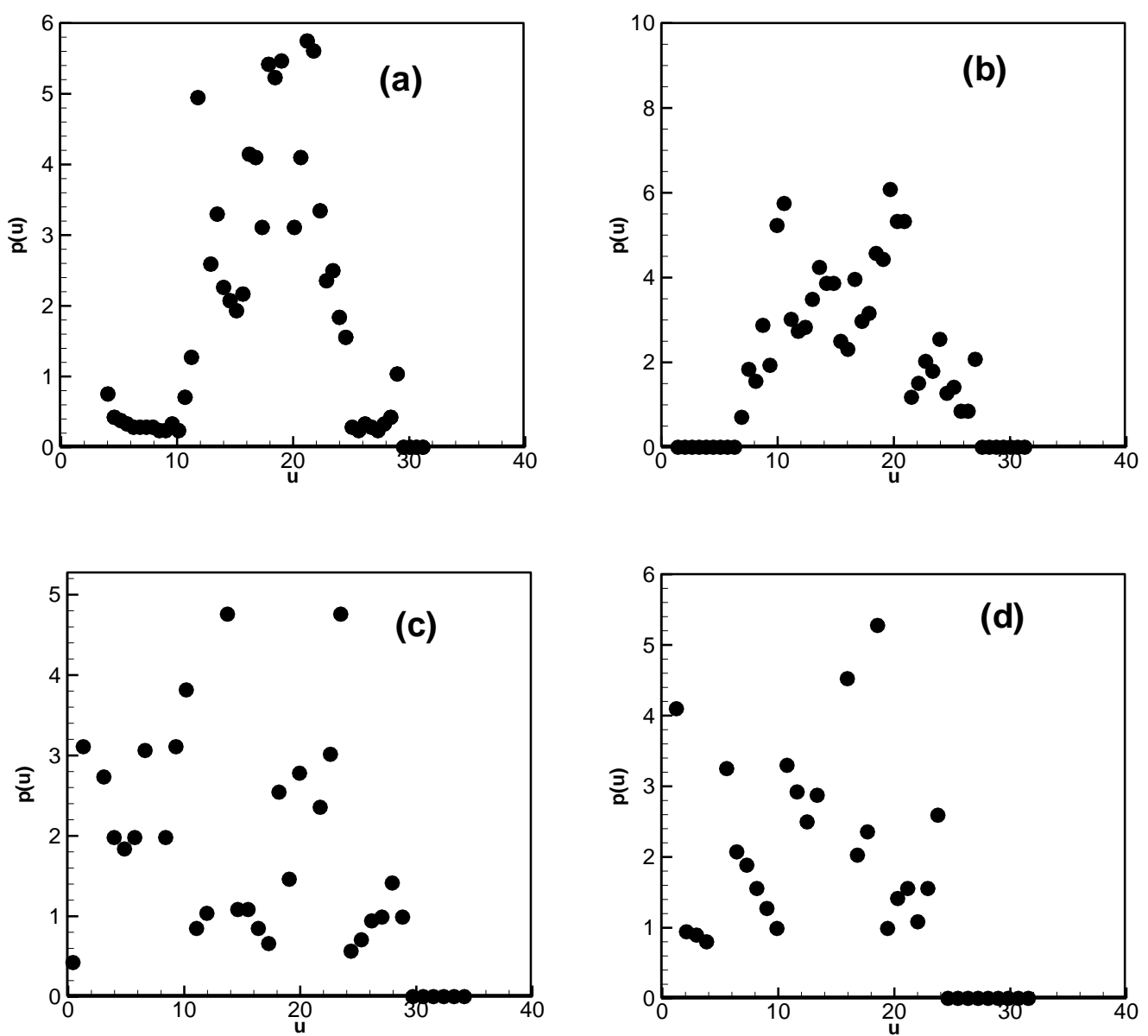

Figure 13. Velocity pdf at $x / D=30$ 

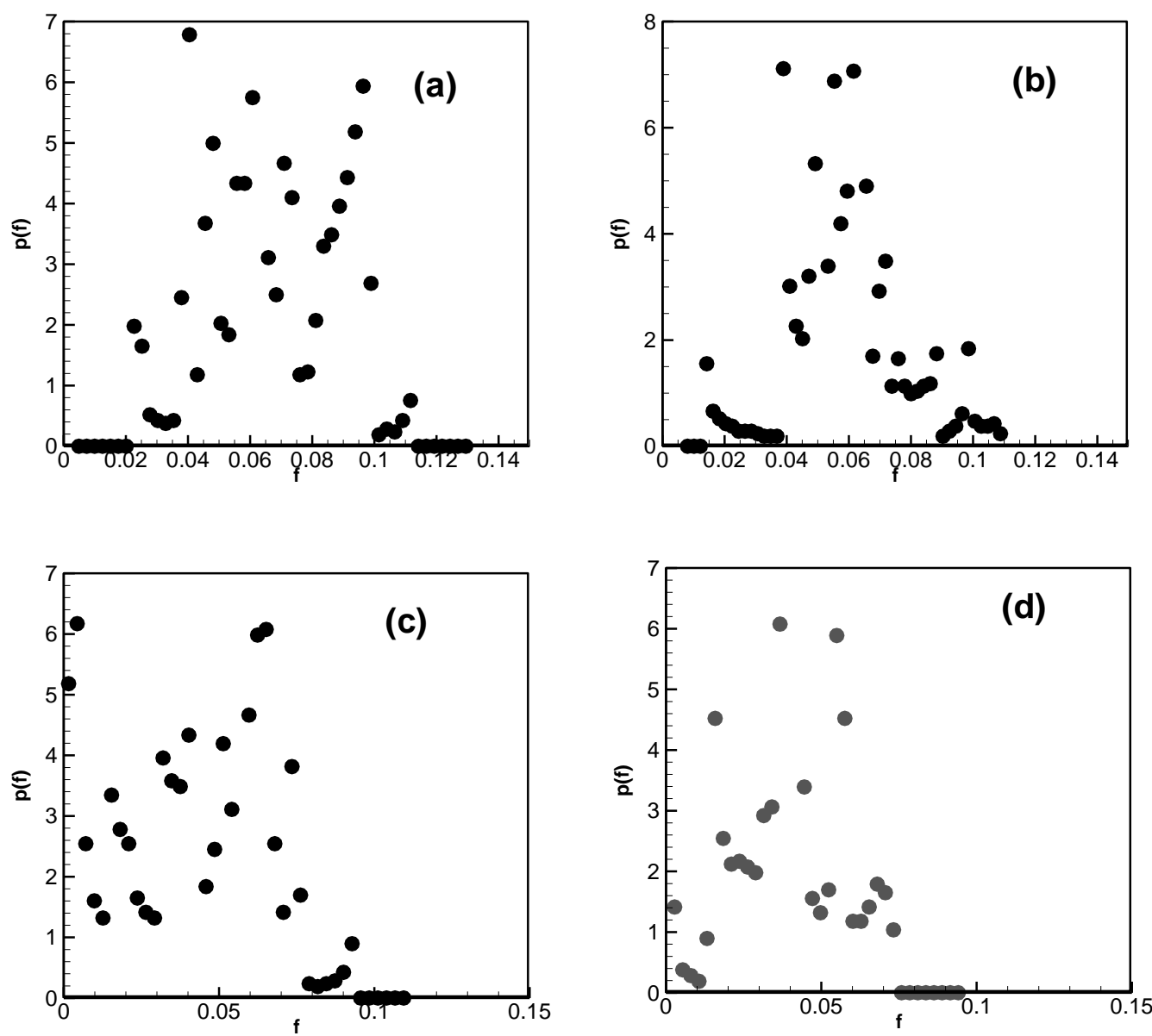

Figure 14. Scalar pdf at $x / D=30$ 

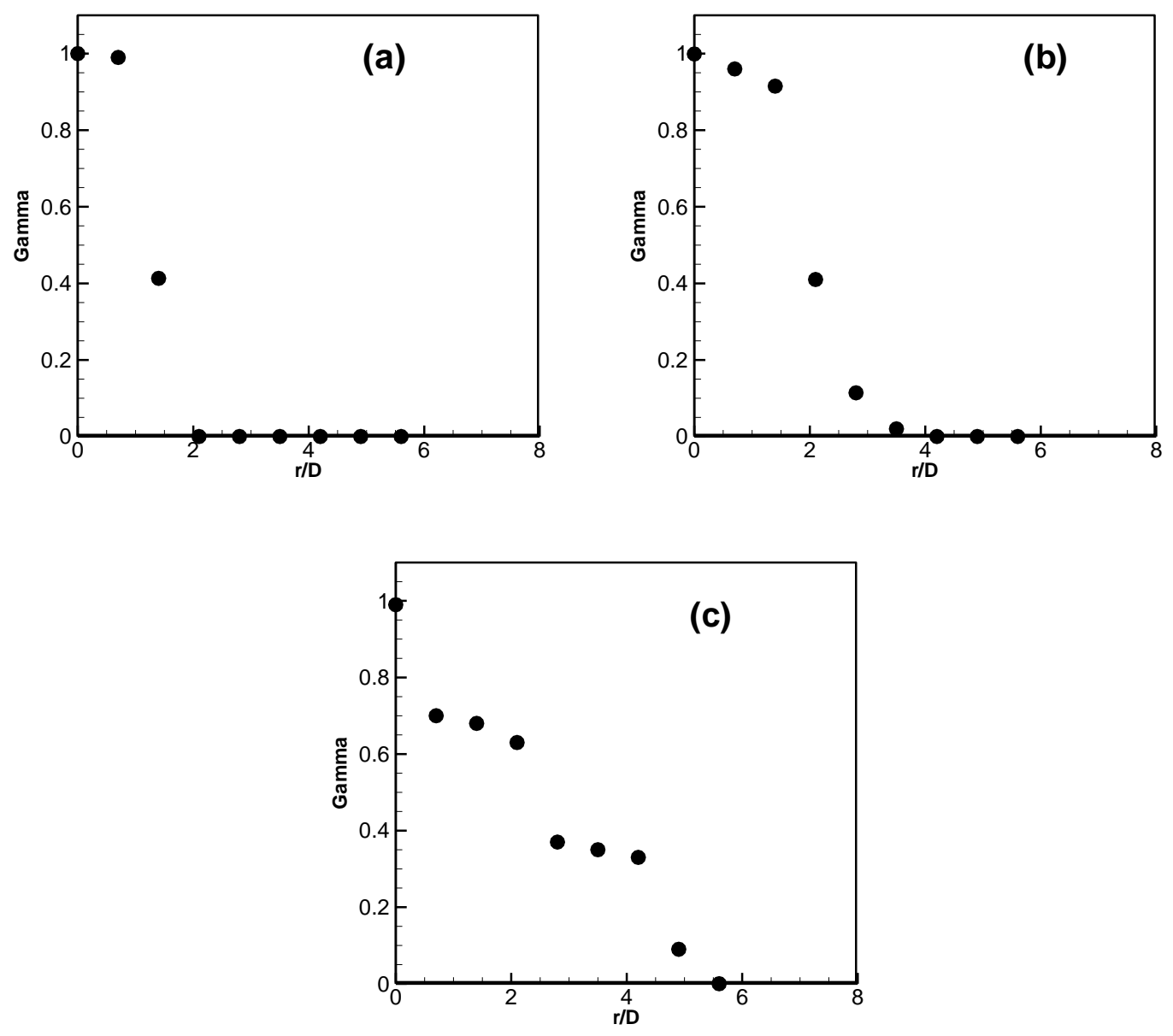

Figure 15. Radial variation of velocity intermittency at (a) $x / D=10$ (b) $x / D=20$ and (c) $x / D=30$ 

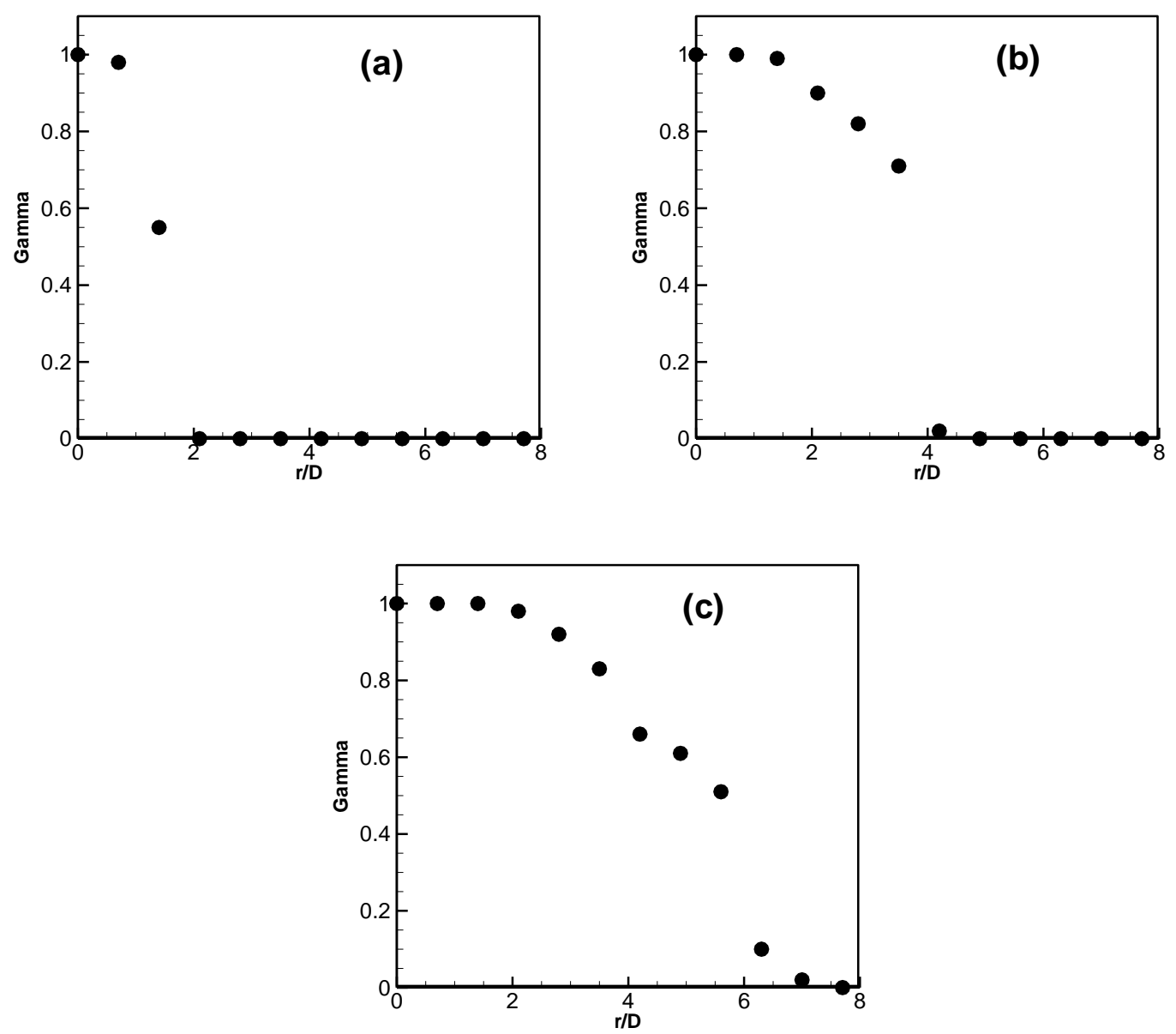

Figure 16. Radial variation of scalar intermittency at (a) $x / D=10$ (b) $x / D=20$ and (c) $\mathrm{x} / \mathrm{D}=30$ 